\section{PO-0334 LENGTH OF STAY CAN BE PREDICTED BY A CLINICAL SCALE MADE IN EMERGENCY DEPARTMENT IN INFANTS WITH ACUTE BRONCHIOLITIS}

${ }^{1} \mathrm{JC}$ Flores-Gonzalez, ${ }^{1} \mathrm{P}$ Comino-Vazquez, ${ }^{1} \mathrm{JJ}$ Perez-Guerrero, 'B Serrano-Moyano, ${ }^{1} \mathrm{P}$ Rodriguez-Campoy, ${ }^{1}$ E Palma-Zambrana, ${ }^{1} \mathrm{MA}$ Matamala-Morillo, ${ }^{1} \mathrm{RM}$ Garcia-Ortega, ${ }^{1} \mathrm{FJ}$ DávilaCorrales, 'L Garcia-Garcia, ${ }^{1} A$ Estalella-Mendoza, ${ }^{1} B$ Grujic, ${ }^{2}$ AM Lechuga-Sancho. ${ }^{1}$ Pediatrics Department, Hospital Universitario Puerta Del Mar, Cádiz, Spain; ${ }^{2}$ Cadiz University, Hospital Universitario Puerta Del Mar, Cádiz, Spain

\subsection{6/archdischild-2014-307384.983}

Backgrounds and aims Wood Downes's modified by Ferres score (WDF) was not designed for the bronchiolitis, but its use has been generalised to evaluate the severity. Our aim is to relate the length of stay and the bronchiolitis's severity at admission, by WDF.

Patient and methods We included the acute bronchiolitis hospitalised during two epidemics (2011-2013). They were classified in mild $(\mathrm{MiB}$; WDF $<4)$, moderated (MB; WDF 4-7) or severe (SB; WDF > 7) according to the scale WDF realised at admission. The mild ones and to the patients without WDF at admission were excluded. The main variable was the length of stay. We registered: age, RSV, sex, previous and during treatment and UCIP's need. Analysis with SPSS 17.0.

Results 208 hospitalised infants were included (mean age of 72,9 days (5-373). Positive RSV in 67,8\%. 90,5\% were MB and $9,5 \%$ SB. Both groups were homogeneous in demographics data and previous admission treatment $(\mathrm{p}>0.05)$. The mean WDF at admission was 5,42 (4-10). During the hospitalisation, they received nebulised bronchodilator in 3\% hypertonic saline solution $(3 \% \mathrm{SSH})(65 \%)$ or $3 \% \mathrm{SSH}(35 \%) .22 \%$ received corticoids and $8,2 \%$ antibiotics. Length of stay: 5,3 days (0-46). The MB had an mean length of stay of 4,8 days and the SB of 13,44 (p $=0.0001)$.

Conclusions WDF's scale has demonstrated, in our sample, to be a good predictor of the length of stay in moderate and severe bronchiolitis. The SB had a statistically significant more length of stay than $\mathrm{MB}$.

\section{PO-0335 DOES THE NEBULIZED 3\% HYPERTONIC SALINE SOLUTION REDUCE ADMISSIONS TO PICU IN ACUTE BRONCHIOLITIS?}

${ }^{1} \mathrm{JC}$ Flores-Gonzalez, ${ }^{1} \mathrm{P}$ Comino-Vazquez, ${ }^{1} \mathrm{P}$ Rodriguez-Campoy, ${ }^{2} \mathrm{G}$ Jimenez-Gómez, ${ }^{1} \mathrm{MA}$ Matamala-Morillo, ${ }^{1}$ RM Garcia-Ortega, ${ }^{1} J J$ Perez-Guerrero, 'L Garcia-Garcia, ${ }^{1} F J$ Dávila-Corrales, ${ }^{1} \mathrm{~B}$ Serrano-Moyano, ${ }^{1} \mathrm{E}$ Palma-Zambrana, ${ }^{3} \mathrm{AM}$ Lechuga-Sancho. ${ }^{1}$ Pediatrics Department, Hospital Universitario Puerta Del Mar, Cádiz, Spain; ${ }^{2}$ Research Unit, Hospital Universitario Puerta Del Mar, Cádiz, Spain; ${ }^{3}$ Cadiz University, Hospital Universitario Puerta Del Mar, Cádiz, Spain

\subsection{6/archdischild-2014-307384.984}

Background and aims Nebulised 3\% hypertonic saline (3\%HSS) decreases length of hospitalisation and rate of admission in infants with moderate bronchiolitis. To describe whether treatment with nebulised 3\%HSS, with or without bronchodilators in acute moderate bronchiolitis, decreases the rate of admission to PICU compared to nebulised saline (FSS) with bronchodilators. Material and methods 389 patients with moderate acute bronchiolitis were admitted, 181 were collected retrospectively (group 0: October 2008 to May 2010) and 208 prospectively (group 1: October 2011 to May 2013 patients). Group 0 received treatment with FSS and group 1 received 3\%HSS. The PICU admission rate was recorded as the main variable.
Secondary variables: overall hospital length of stay (LOS), stay at PICU, need for mechanical ventilation (MV) and its duration. The collected variables were analysed using Chi - square and nonparametric tests.

Results Demographic data and clinical data were similar in both groups ( $p>0.05)$ : mean age, gender male, RSV positive, corticosteroids or antibiotics use. LOS was longer in group $0(\mathrm{p}=$ 0.020 ). PICU admission was $17.8 \%$ vs $12.5 \%, \mathrm{p}=0.146$, stay PICU: 5.91 days vs 3.76 days, $\mathrm{p}=0.859), \mathrm{VM}$ rate in PICU was $25 \%$ vs $24 \%, p=0.931$ and VM duration of PICU was 7.63 days vs 7.17 days.

Conclusions Patients treated with $3 \% \mathrm{SSH}$ showed the same PICU admission rate and stay. The nebulised 3\%SSH does not decrease the PICU admission. Overall hospital LOS was longer in FSS group.

\section{PO-0336 NECROTIZING ENTEROCOLITIS (NEC) AND FOCAL INTESTINAL PERFORATION (FIP) TREATMENT IN A SMALL SINGLE-CENTRE NEONATOLOGY}

A Cazzuffi, E Cesca, A Franchella. Maternity and Childhood, Pediatric Surgery, Ferrara, Italy

\subsection{6/archdischild-2014-307384.985}

Background and aim Necrotizing enterocolitis (NEC) and focal intestinalperforation (FIP) are the mostimportant acquired intestinal diseases in preterm infants with very low birthweight (VLBW). Aim of the study was to evaluate surgical procedures, short-termoutcome and survival of ELBW infants with NEC and FIP in a single-centre study.

Methods ELBW infantsaffected by NEC and FIP and surgically treated in the Neonatology of theUniversity-Hospital of Ferrara from 2000 and 2013 wereretrospectively analysed.

Results Sixteen ELBW infants underwent surgery, 6 because of FIP and 10 for NEC. Three infants in the FIP group were treated with primary laparotomy and 5 withperitoneal drainage (PD). In the NEC group, 4/10 infants were treated with PD,the others with laparotomy. PD was used for unstable patients and was nearly always followed bysecondary laparotomy after stabilisation. In our series just one patient healedwithout further procedures after PD. Five of 16 (12.5\%) surgically treated ELBWinfants died. Complications occurred in 3/16 (18.75\%) infants: one patientunderwent re-laparotomy for NEC recurrence and 2 were treated for intestinal obstruction after stomaclosure.

\section{PO-0337 WITHDRAWN}

\section{PO-0338 EVALUATION OF SUBLINGUAL MICROCIRCULATION IN CRITICALLY ILL CHILDREN USING SIDESTREAM DARK FIELD MICROSCOPY: CORRELATION WITH OTHER ROUTINELY USED VARIABLES}

R Gonzalez Cortes, J Urbano, MJ Solana, J López, SN Fernandez, B Toledo, J Lopez-Herce. Pediatric Intensive Care Unit, Gregorio Marañón University Hospital, Madrid, Spain

\subsection{6/archdischild-2014-307384.986}

Background and aims Microcirculation is usually evaluated by indirect means in critically ill children. The aim of this study is to evaluate if there is correlation between microcirculation 